\title{
Correspondence
}

\section{Bortezomib, Lenalidomide and Dexamethasone as Induction Therapy Prior to Autologous Transplantation in Multiple Myeloma: The More Is Likely the Better}

\author{
Mohamad Mohty ${ }^{1, *}$, Florent Malard ${ }^{1,(1)}$, Ali Bazarbachi ${ }^{2,3}$ \\ ${ }^{1}$ Sorbonne Universités, INSERM, Centre de Recherche Saint-Antoine (CRSA), Hematology Department, AP-HP, Saint Antoine Hospital, Paris, France \\ ${ }^{2}$ Bone Marrow Transplantation Program, Department of Internal Medicine, American University of Beirut Medical Center, Beirut, Lebanon \\ ${ }^{3}$ Department of Anatomy, Cell Biology, and Physiological Sciences, American University of Beirut, Beirut, Lebanon
}

\section{ARTICLE INFO}

Article History

Received 24 Jan 2020

Accepted 07 May 2020

(C) 2020 International Academy for Clinical Hematology. Publishing services by Atlantis Press International B.V. This is an open access article distributed under the CC BY-NC 4.0 license (http://creativecommons.org/licenses/by-nc/4.0/).

The autologous hematopoietic cell transplantation (auto-HCT) procedure for multiple myeloma (MM) comprises several key steps: induction, hematopoietic cell mobilization, high-dose chemotherapy followed by hematopoietic cell infusion and posttransplant therapy (including maintenance therapy with or without prior consolidation). For almost 3 decades, the standard induction regimen prior to auto-HCT consisted of 3-4 cycles of vincristine, adriamycin and dexamethasone (historical VAD regimen). The IFM2005-01 randomized trial was the first trial to show the superiority of a novel-agent-based induction (namely bortezomib and dexamethasone, VD) over VAD [1]. The VD regimen served as a backbone to develop triplet induction regimens such as VTD (bortezomib, thalidomide, dexamethasone) [2] or VCD (bortezomib, cyclophosphamide, dexamethasone), both of which were proven to be superior to doublets such as VD or TD (thalidomide and dexamethasone) [2,3]. Of note, another IFM randomized trial could show a slight superiority of VTD over VCD in terms of response rate [4]. Interestingly, none of the aforementioned triplet regimens explored the issue of the number of cycles, and investigators continued to use the classical 3-4 cycles based on the historical VAD experience. However, one must admit, that given the potential serious toxicity of agents such as thalidomide (nonreversible peripheral neuropathy) or cyclophosphamide (cytopenia and its corollary of infectious complications), the issue of the number of cycles of induction prior to auto-HCT was not deemed to be very important by most investigators, especially given the capacity to deliver 2 or 3 cycles of consolidation to deepen the response rate after auto-HCT.

*Corresponding author. Email: mohamad.mohty@inserm.fr Peer review under responsibility of the International Academy for Clinical Hematology
The VRD regimen (bortezomib, lenalidomide, dexamethasone) was initially developed in the relapsed MM setting [5] and proved rapidly to be highly effective with a remarkable safety profile, especially a very low rate of severe peripheral neuropathy when combined to subcutaneous bortezomib. Very rapidly, VRD moved into the frontline setting, and was viewed by most experts as the "standard" induction regimen prior to auto-HCT, since it combines the efficacy of VTD without its added toxicity. The latter positive ratio of efficacy and safety was clearly shown in the trial by Rosinol et al. [6]. In addition, given its good tolerance and feasibility, Rosinol et al. took this regimen a step further, and administered VRD in up to 6 cycles (in contrast to the common historical dogma of 3-4 cycles). The former strategy proved to be highly effective as it allowed for a continuous improvement in the disease response, including achievement of measurable residual disease (MRD) negativity in a substantial number of patients before auto-HCT [6]. These results are of utmost importance, because a deep MRD status is increasingly considered a major goal of MM therapy, because it probably serves as a surrogate marker for improved survival. Overall, the findings from this large Spanish trial will be highly influential and will encourage many investigators to opt for a "longer" induction phase of up to 6 cycles, toxicity allowing.

However, MM therapy is a rapidly evolving field $[7,8]$, and one may wonder how such a "long" induction phase would compare to a quadruplet regimen-based induction administered over a shorter period of time? In this regard, the recent CASSIOPEIA international randomized trial evaluated whether the addition of daratumumab to VTD (Dara-VTD) before (induction) and after auto-HCT (consolidation) would improve the stringent complete response rate in patients with newly diagnosed MM [9]. The 
latter study showed the clinical benefit of Dara-VTD given in up to 4 cycles as a major regimen for induction in transplant-eligible patients with newly diagnosed MM. As a matter of fact, and given the impressive results of Dara-VTD, it is possible that a Dara-VRDbased induction regimen (currently being tested in prospective trials) will also probably show a significant benefit in this arena. Nevertheless, one should not ignore issues related to cost and access to such quadruplet regimens, though they can also challenge the whole concept of auto-HCT if shown to be feasible over a long period (e.g. a quadruplet regimen administered for one year) and able to induce deep MRD negativity [10]. While waiting for the results of such trials, auto-HCT preceded by a "long" induction with VRD will likely remain a standard of care for many years in many patients across the globe.

\section{CONFLICT OF INTERESTS}

FM reports lecture honoraria from Therakos/Mallinckrodt, Janssen, Keocyte, Sanofi, JAZZ pharmaceutical and Astellas, all outside the submitted work. MM reports grants and lecture honoraria from Janssen, Sanofi, Maat Pharma and JAZZ pharmaceutical, lecture honoraria from Celgene, Amgen, BMS, Takeda, and Pfizer, grants from Roche. The other authors declare no competing financial interests.

\section{AUTHORS' CONTRIBUTIONS}

All authors contributed substantially to the conception, writing, critical review and final approval of the manuscript.

\section{ACKNOWLEDGMENTS}

The authors acknowledge the Association for Training, Education and Research in Hematology, Immunology and Transplantation for the generous and continuous support of the research work. We thank our nursing staff for offering the best care to our patients.

\section{REFERENCES}

[1] Harousseau, JL, Attal, M, Avet-Loiseau, H, et al. Bortezomib plus dexamethasone is superior to vincristine plus doxorubicin plus dexamethasone as induction treatment prior to autologous stem-cell transplantation in newly diagnosed multiple myeloma: results of the IFM 2005-01 phase III trial. J Clin Oncol 2010;28;4621-9.

[2] Cavo, M, Tacchetti, P, Patriarca, F, et al. Bortezomib with thalidomide plus dexamethasone compared with thalidomide plus dexamethasone as induction therapy before, and consolidation therapy after, double autologous stem-cell transplantation in newly diagnosed multiple myeloma: a randomised phase 3 study. Lancet 2010;376;2075-85.

[3] Moreau, P, Avet-Loiseau, H, Facon, T, et al. Bortezomib plus dexamethasone versus reduced-dose bortezomib, thalidomide plus dexamethasone as induction treatment before autologous stem cell transplantation in newly diagnosed multiple myeloma. Blood 2011;118;5752-8, quiz 982.

[4] Moreau, P, Hulin, C, Macro, M, et al. VTD is superior to VCD prior to intensive therapy in multiple myeloma: results of the prospective IFM2013-04 trial. Blood 2016;127;2569-74.

[5] Richardson, PG, Weller, E, Lonial, S, et al. Lenalidomide, bortezomib, and dexamethasone combination therapy in patients with newly diagnosed multiple myeloma. Blood 2010;116;679-86.

[6] Rosiñol, L, Oriol, A, Rios, R, et al. Bortezomib, lenalidomide, and dexamethasone as induction therapy prior to autologous transplant in multiple myeloma. Blood 2019;134;1337-45.

[7] Bonello, F, Cetani, G, Bertamini, L, et al. Moving toward continuous therapy in multiple myeloma. Clin Hematol Int 2019;1; 189-200.

[8] Szabo, AG, Fladeland Iversen, K, Möller, S, et al. The clinical course of multiple myeloma in the era of Novel agents: a retrospective, single-center, real-world study. Clin Hematol Int 2019;1; 220-8.

[9] Moreau, P, Attal, M, Hulin, C, et al. Bortezomib, thalidomide, and dexamethasone with or without daratumumab before and after autologous stem-cell transplantation for newly diagnosed multiple myeloma (CASSIOPEIA): a randomised, open-label, phase 3 study. Lancet 2019;394;29-38.

[10] Harousseau, JL, Avet-Loiseau, H. Minimal residual disease negativity is a new end point of myeloma therapy. J Clin Oncol 2017;35;2863-5. 